\title{
Open Photoacoustic Cell Technique as a Tool for Thermal and Thermo-Mechanical Characterization of Teeth and Their Restorative Materials ${ }^{1}$
}

\author{
J. L. Pichardo-Molina, ${ }^{2,3}$ G. Gutiérrez-Juárez, ${ }^{4}$ R. Huerta-Franco, ${ }^{5}$ \\ M. Vargas-Luna, ${ }^{4}$ P. Cholico, ${ }^{6}$ and J. J. Alvarado-Gil ${ }^{7}$
}

\begin{abstract}
The thermal diffusivity and thermal expansion coefficient of teeth and three of their most common restorative materials (Amalgam Phase Alloy, Ionomer Fuji II LC, and Resin 3MFPITEK Lutine ${ }^{\mathrm{TM}} \mathrm{Z} 250$ ) were studied by means of the open photoacoustic technique. These results were then used as a basis for the theoretical simulation of the photothermal process taking place as a consequence of modulated illumination of a two-layer system formed by the tooth and the restorative material. The model accounts for the coupling of thermal waves and thermoelastic vibration in the two-layer system.
\end{abstract}

KEY WORDS: photoacoustic technique; teeth; thermal properties; tooth restorative materials.

\section{INTRODUCTION}

Although health care is one of the most important issues in our society, attention is frequently paid to the body's esthetic appearance instead.

\footnotetext{
${ }^{1}$ Paper presented at the Fifteenth Symposium on Thermophysical Properties, June 22-27, 2003, Boulder, Colorado 80303

${ }^{2}$ Centro de Investigaciones en Óptica A. C., Loma del Bosque 115, Col. Loma del Campestre, C. P. 37150 León Gto., México.

${ }^{3}$ To whom correspondence should be addressed. E-mail: jpichardo@cio.mx

${ }^{4}$ Instituto de Física de la Universidad de Guanajuato, A. P. E-143, C. P. 37000, León Gto, México.

${ }^{5}$ Instituto de Investigaciones sobre el Trabajo, Universidad de Guanajuato, Avenida Eugenio Garza Sada No. 572, Lomas del Campestre, 37150 León Gto., México.

${ }^{6}$ Dpto. de Clínicas Odontológicas Integrales, Universidad de Guadalajara, José Maria Echaury y Juan Díaz Covarrubias, C. P. 44280 Guadalajara, Jal. México.

${ }^{7}$ Departamento de Física Aplicada, CINVESTAV-Unidad Mérida, Carretera Antigua a progreso, Km. 6, Cordemex, Mérida, 97310 Yucatán, México.
} 
In recent years, the dentistry market has developed new materials to satisfy the health and aesthetic requirements of their customers. However, the first generation of these new composite materials was limited by low wear resistance to abrasion, moderate diversity of colors, increased polymerization shrinkage, low flexural strength and modulus of elasticity, and finally relatively high incidence of fracture. Newer formulations offer better advantages overcoming these difficulties and ensuring high acceptance for tooth restoration. New resins, amalgams, and cements are currently used; however, the thermal and thermo-mechanical information needed for their proper application is not always available.

Taking into account only the health aspect, the market of the dentistry is mainly focused on durability and comfort. One of the most important aspects is the behavior of the restored teeth when exposed to heating. Matching of thermal, mechanical, and thermo-mechanical properties between teeth and their restorative materials, is one of the main concerns in this field. The study of these properties in new composite materials and comparisons of data to those for teeth and the traditional amalgam is of major importance for their use in teeth restoration.

Several standard techniques have been used to determine thermal and thermo-mechanical properties of different kind of materials. Some of the most used are as follows: X-ray, mechanical dilatometer, laser interferometric dilatometer, laser flash (which is based on the modified Parker method), Ånstrong method, and phase-lag methods [1-4]. The first three techniques mentioned above are used to determine the linear thermal expansion coefficient and the last three are use to determine thermal diffusivity; therefore, to provide thermal and thermo-mechanical characterization, it is necessary to combine at least two of these techniques. Some of these techniques require a large amount of material to assess their characterization.

Photoacoustic (PA) and related photothermal techniques have been used in a wide variety of fields for simultaneous thermal and thermomechanical characterization using a minimum amount of material. The PA technique considers directly the heat and pressure waves, generated in a sample, following the absorption of pulsed or modulated electromagnetic radiation. In the conventional PA experimental arrangement using a microphone, the sample is enclosed in an air-tight chamber and exposed to modulated light. Such periodic heating of the sample will produce the pressure in the chamber that oscillates at the same modulation frequency; the pressure is detected with the microphone coupled to the cell. The resulting PA signal depends not only on the amount of the heat in the sample, but also on how the same heat diffuses through the sample as well as its surroundings. The open photoacoustic cell (OPC) concept was 
developed as a suitable method to determine thermo-mechanical properties of solids by considering a bending phenomenon due to the temperature profile across the thickness of the sample [5]. It has been shown that when the OPC method is applied to layered systems [6], one can obtain the thermal diffusivity, $\alpha_{s}$, and the thermal expansion coefficient, $\alpha_{T}$, of the individual layers separating components of the systems. On the other hand, a tooth that needs to be restored will undergo significant temperature fluctuations. Therefore, predictions of the thermal behavior of the layered material, based on the properties of each layer, are also important.

In composite materials, it is very important to understand the coupling of the thermal and thermo-mechanical phenomena, because this information could be used to characterize the behavior of this kind of materials. In this work, the thermal and thermo-mechanical characterization of teeth and their restorative materials using the OPC technique was performed. The experimental results were used to perform a theoretical study of the photothermal effects in the layered tooth-restorative material system.

\section{EXPERIMENT AND THEORY}

The OPC technique makes use of the heat transmission configuration [6-8], with the sample mounted (fixed with vacuum grease) directly to the front opening (circular hole, $3 \mathrm{~mm}$ in diameter) of an electret microphone. The front microphone chamber adjacent to the metallized face of the diaphragm is a cylinder of $7 \mathrm{~mm}$ diameter and $1 \mathrm{~mm}$ length (Fig. 1).

The PA signal is generated by illuminating the sample with a modulated light beam. The beam of a $70 \mathrm{~mW}$ He-Ne laser (Melles Griot 05-LHP-928), modulated by a mechanical chopper (Stanford Research Systems SR540), was focused onto the sample. The signal from the microphone was connected to a lock-in amplifier (Stanford Research Systems SR850). Both the signal amplitude and phase were recorded as a function of modulation frequency.

By fitting the experimental data for the amplitude phase of the OPC signal (as a function of the modulation frequency with Eq. (1)), one can obtain $\alpha_{\mathrm{s}}$ and $\alpha_{\mathrm{T}}$.

\subsection{Homogeneous Layers}

According to the transmission configuration model that takes into account the thermal diffusion and thermo-elastic bending of an opaque sample, the output voltage of the microphone is given by [5] 

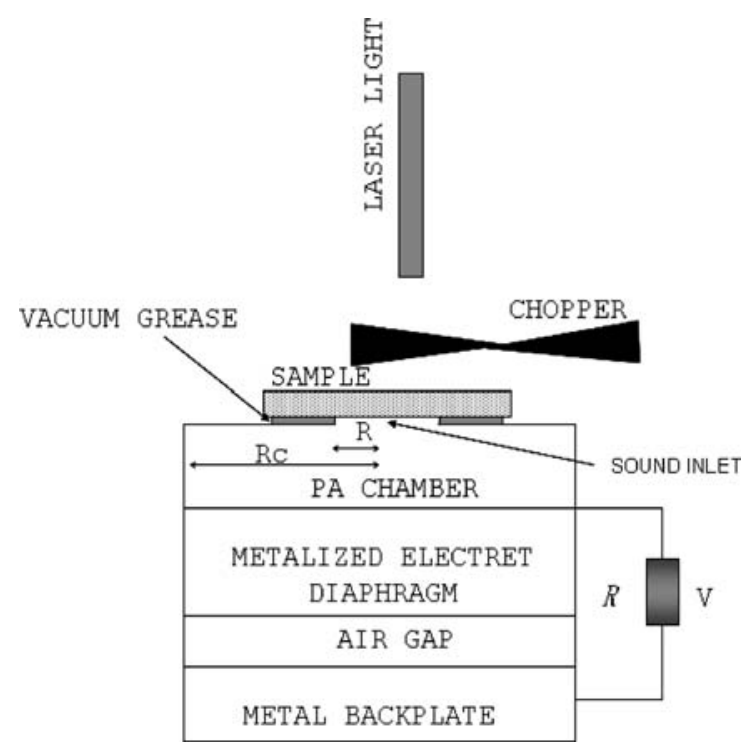

Fig. 1. Experimental setup.

$$
\begin{aligned}
V_{\mathrm{opc}}= & V_{0}\left[\frac{j \omega \tau_{\mathrm{E}} e^{-j \omega t}}{1+j \omega \tau_{\mathrm{E}}} \frac{I_{0}}{T_{0} l_{\mathrm{g}} \sigma_{\mathrm{g}} k_{\mathrm{s}} \sigma_{\mathrm{s}}}\right] \\
& \times\left[\frac{1}{\sinh \left(l_{\mathrm{s}} \sigma_{\mathrm{s}}\right)}-\frac{3 R^{4} \alpha_{\mathrm{T}} T_{0}}{2 R_{\mathrm{c}}^{2} l_{\mathrm{s}}^{2}} \sqrt{\frac{\alpha_{\mathrm{s}}}{\alpha_{\mathrm{T}}}} \frac{\left(l_{\mathrm{s}} \sigma_{\mathrm{s}} / 2\right) \sinh \left(l_{\mathrm{s}} \sigma_{\mathrm{s}}\right)-\cosh \left(l_{\mathrm{s}} \sigma_{\mathrm{s}}\right)+1}{l_{\mathrm{s}} \sigma_{\mathrm{s}} \sinh \left(l_{\mathrm{s}} \sigma_{\mathrm{s}}\right)}\right],
\end{aligned}
$$

where $I_{0}$ is the incident radiation intensity and $\omega=2 \pi f$ (with $f$ being the modulation frequency). Furthermore, $V_{0}$ is a constant depending on the microphone characteristics; $\tau_{\mathrm{E}}$ is the microphone response time; $j=\sqrt{-1}$; $T_{0}$ refers to the room temperature; $R_{\mathrm{c}}$ is the radius of the chamber in front of the microphone diaphragm $\left(R_{\mathrm{c}}=3.5 \mathrm{~mm}\right)$; and $R$ is the radius of the front opening $(R=1.5 \mathrm{~mm})$. The symbols $l_{i}, k_{i}, \alpha_{i}$, and $\sigma_{i}$ are used for the thickness, thermal conductivity, thermal diffusivity, and complex thermal diffusion coefficient $\left(\sigma_{i}=(1+j)\left(\pi f / \alpha_{i}\right)^{1 / 2}\right)$, respectively; subscript $i$ refers to either the sample $(s)$ or air $(g)$. Finally, $\alpha_{\mathrm{T}}$ is the linear thermal expansion coefficient.

\subsection{Homogeneous Two-Layer System}

In order to obtain information about the behavior of the twolayer system (teeth and restorative dental material), simulations of the PA signal were performed. The two-layer model used here accounts for 
thermal diffusion and thermo-elastic vibration, and assumes a perfect match between the thermal waves and thermo-mechanical vibration at the interface of both layers. Moreover, it is assumed that the mechanical properties (Young's modulus and Poisson ratio) of both materials are comparable. An extensive analysis [9] of such a model includes the extrapolation of the model developed by Rousset and Lepoutre [10]. The PA signal is given by the following expression:

$$
V_{\mathrm{opc}}=V_{0}\left[\frac{j \omega \tau_{\mathrm{E}}}{1+j \omega \tau_{\mathrm{E}}}\right] \times \frac{\delta P_{\text {Diff }}+\delta P_{\text {Therm }}}{\gamma P_{0}} e^{i \omega t},
$$

where $\delta P_{\text {Diff }}$ and $\delta P_{\text {Therm }}$ are the contributions of thermal diffusion and thermoelastic bending, respectively, and are given by

$$
\delta P_{\text {Diff }}=-\frac{I_{0} \gamma P_{0}}{l_{\mathrm{g}} \sigma_{\mathrm{g}} k_{1} \sigma_{1}(1+s)}\left[\frac{1}{\Sigma+\eta \mathrm{X}}\right]
$$

and

$$
\begin{aligned}
\delta P_{\mathrm{Therm}}= & -\frac{R^{4} I_{0}}{8 k_{1} \sigma_{1} R_{\mathrm{c}}^{2} l_{\mathrm{g}}}\left\{\frac{6}{l^{2}}\left[\alpha_{\mathrm{T} 1}\left(\frac{X_{2} \Sigma_{1}+s\left(X_{1}-1\right) \Sigma_{1}}{\sigma_{1}}\right)+\alpha_{\mathrm{T} 2} \frac{\Sigma_{2}}{\sigma_{2}}\right]\right. \\
& -\frac{12}{l^{3}}\left[\alpha_{\mathrm{T} 1}\left(\left(X_{1}-1\right) \frac{X_{2}}{\sigma_{1}^{2}}+s\left(\Sigma_{2}-\sigma_{1} l_{1}\right) \frac{\Sigma_{2}}{\sigma_{1}^{2}}\right)\right. \\
& \left.\left.+\alpha_{\mathrm{T} 2}\left(\frac{X_{2}+l_{1} \sigma_{2} \Sigma_{2}-1}{\sigma_{2}^{2}}\right)\right]\right\}\left(X_{2} \Sigma_{1}+s X_{1} \Sigma_{2}\right)^{-1},
\end{aligned}
$$

where $\Sigma=\sinh \left(\sigma_{1} l_{1}+\sigma_{2} l_{2}\right), \mathrm{X}=\sinh \left(\sigma_{1} l_{1}-\sigma_{2} l_{2}\right), \eta=\frac{1-s}{1+s}, \mathrm{X}_{i}=\cosh \left(\sigma_{i} l_{i}\right)$, $\Sigma_{i}=\sinh \left(\sigma_{i} l_{i}\right)$ and the subscripts, $i=1,2, g$ refer to layers 1 , and 2 , and to a surrounding gas, respectively, and finally $\gamma=c_{\mathrm{p}} / c_{\mathrm{v}}$ is the ratio of heat capacities at constant pressure and volume of air.

\subsection{Materials}

The sample materials were obtained from molars of Holstein-Friesian cow (samples C1-C4) and from the third molar of a human subject (sample H1). Thin samples of teeth, around $1 \mathrm{~mm}$ thickness, were sliced with a diamond saw. The samples were prepared as follows: preliminary mechanical polishing was carried out with sandpaper (ultra-fine 600 grit) to obtain samples with a thickness of $500 \mu \mathrm{m}$. To obtain the final thickness, an electromechanical system was used for each sample. This system consists of two horizontal flat plates; the sample was glued to the upper one, and the polisher (aluminum oxide of 25 and $9 \mu \mathrm{m}$ of grit) was placed on the 
lower one. The sample and the polisher were in contact, and by mechanical movement, the samples were polished to get the final thickness. Finally, the samples were measured with a standard micrometer (Mitutoyo).

In order to obtain optically opaque samples in the region of the $\mathrm{He}-\mathrm{Ne}$ laser wavelength, teeth samples were painted with a metallic paint. This paint layer had about $10 \mu \mathrm{m}$ thickness, and it did not affect the measurements (this was verified by theoretical simulation).

Three different samples of restorative materials were prepared: amalgam of $366 \mu \mathrm{m}$ (Phase Alloy), ionomer sample of $267 \mu \mathrm{m}$ (Fuji II LC), and resin sample of $366 \mu \mathrm{m}$ (3MFPITEK Lutine ${ }^{\mathrm{TM}} \mathrm{Z} 250$ ); these samples were labeled Am, Io, and Re, respectively. For the ionomer and resin samples, instead of paint layer, an aluminum foil of $10 \mu \mathrm{m}$ was glued using a thermal paste to get optically opaque samples. Even if the mechanical properties (Young's modulus and Poisson ratio) have similar values for both materials, the thermal compatibility should be carefully analyzed.

\section{RESULTS}

\subsection{Homogeneous Materials}

Samples of human and cow teeth were characterized thermally and mechanically using modulation frequencies from 40 to $400 \mathrm{~Hz}$. This frequency interval was chosen because within this range neither of these contributions dominates. At frequencies below $40 \mathrm{~Hz}$, the thermal diffusion starts to prevail; on the other hand, at frequencies exceeding $400 \mathrm{~Hz}$, it is the thermo-elastic phenomenon that dominates. Figure 2 shows the experimentally obtained signals (full circles) as a function of the modulation frequency for $\mathrm{H} 1$ and $\mathrm{C} 3$ samples. The continuous line is the best fit to Eq. (1). The same procedure was carried out for all other samples. When studying dental restorative materials, the range of modulation frequencies was expanded $(40-800 \mathrm{~Hz})$. Figure 3 shows the PA signals (full circles) obtained for dental materials; the continuous line represents the best fit to Eq. (1). The values of $\alpha_{\mathrm{s}}$ and $\alpha_{\mathrm{T}}$ obtained for all samples are displayed in Table I.

The values of the thermal diffusivity, determined by the OPC technique, are of the same order of magnitude as values reported in the literature. However, C2, C3, and C4 samples have low values of the linear thermal expansion coefficient compared with values from the literature for human teeth. $\mathrm{H} 1$ and $\mathrm{C} 1$ samples have larger values of thermal diffusivity compared with those from the literature.

It is important to mention that teeth samples were obtained from cross-sections at different depth levels. Therefore, for the $\mathrm{C} 1$ sample, the 


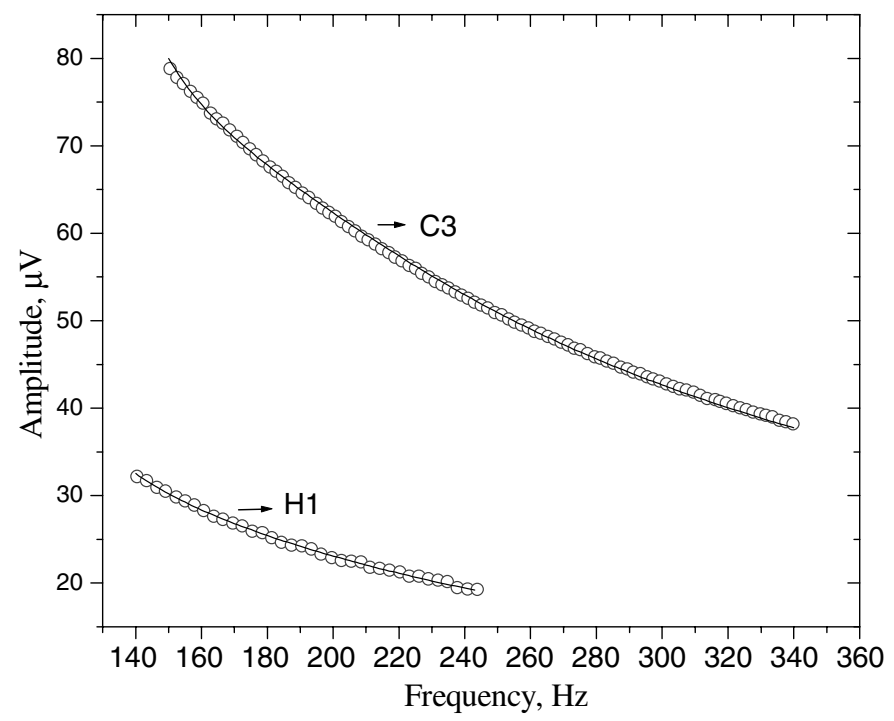

Fig. 2. Amplitude of the PA signal obtained from $H 1$ and $C 3$ samples, plotted as a function of the modulation frequency. Full circles represent the experimental data, and the continuous line is the best fit to Eq. (1).

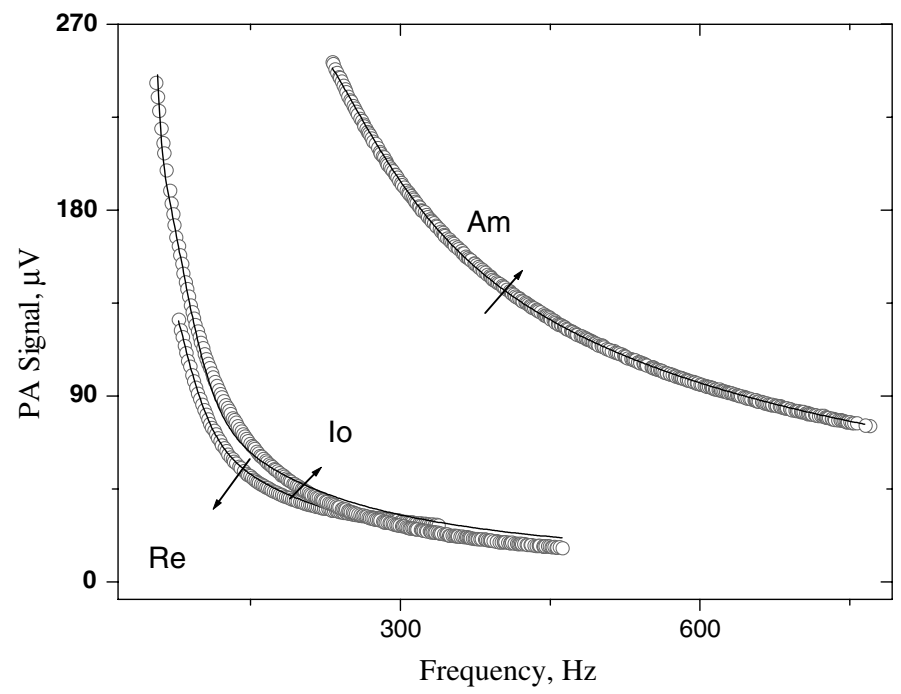

Fig. 3. Amplitude of the PA signal obtained from Am, Re and Io materials plotted as a function of the modulation frequency. Experimental data are represented by empty circles while the continuous line represents the best fit to Eq. (1) in each case. 
content of enamel is greater than for the other $\mathrm{C}$ samples, which correspond to cross sections from the internal layers of the teeth with a larger amount of dentine. On the other hand, a tooth is composed primarily of collagen, water, and hydroxyapatite. The percentage of hydroxyapatite in the enamel and dentin are 95 and $70 \%$, respectively. The thermal expansion coefficient of hydroxyapatite is around $10 \times 10^{-6} \mathrm{~K}^{-1}$, which is a value closer to that for the $\mathrm{Cl}$ sample. Also, it is important to mention that the reported values came from human teeth, whereas the samples $\mathrm{C} 1$, $\mathrm{C} 2, \mathrm{C} 3$, and $\mathrm{C} 4$ belong to cow teeth.

\subsection{Two-Layer Model}

Figure 4 shows theoretical results for the amplitude of the PA signal from teeth and two-layer systems composed of the teeth and a restorative material (Am, Re, and Io). In each case the total thickness of the sample was $600 \mu \mathrm{m}$. In the two-layer case, the thickness of the dental material was $500 \mu \mathrm{m}$ compared to a thickness of $100 \mu \mathrm{m}$ of the restorative material. Thermal conductivity values $k_{\mathrm{H}}=0.0090 \mathrm{~W} \cdot \mathrm{cm}^{-1} \cdot \mathrm{K}^{-1}$ (enamel), $k_{H}=0.0056 \mathrm{~W} \cdot \mathrm{cm}^{-1} \cdot \mathrm{K}^{-1}$ (dentin), $k_{\mathrm{A}}=0.225 \mathrm{~W} \cdot \mathrm{cm}^{-1} \cdot \mathrm{K}^{-1}$, $k_{\mathrm{R}}=0.0015 \mathrm{~W} \cdot \mathrm{cm}^{-1} \cdot \mathrm{K}^{-1}$, and $k_{\mathrm{I}}=0.006 \mathrm{~W} \cdot \mathrm{cm}^{-1} \cdot \mathrm{K}^{-1}$ were taken from the literature [11,12], for the human teeth (enamel and dentin), Am, Re, and Io, respectively, for all materials. The thermal diffusivity and linear expansion coefficient were measured for all materials, and the values obtained are reported in Table I.

Table I. Thermal Diffusivity and Linear Thermal Expansion Coefficient of Teeth and Restorative Materials.

\begin{tabular}{ccccrc}
\hline Sample & $\begin{array}{c}l_{\mathrm{s}} \\
(\mu \mathrm{m})\end{array}$ & $\begin{array}{c}\alpha_{\mathrm{s}} \text { (measured) } \\
\left(10^{-2} \mathrm{~cm}^{2} \mathrm{~s}^{-1}\right)\end{array}$ & $\begin{array}{c}\alpha_{\mathrm{s}} \text { (reported) } \\
\left(10^{-2} \mathrm{~cm}^{2} \mathrm{~s}^{-1}\right)\end{array}$ & $\begin{array}{c}\alpha_{\mathrm{T}} \text { (measured) } \\
\left(10^{-6} \mathrm{~K}^{-1}\right)\end{array}$ & $\begin{array}{c}\alpha_{\mathrm{T}} \text { (reported) } \\
\left(10^{-6} \mathrm{~K}^{-1}\right)\end{array}$ \\
\hline $\mathrm{H} 1$ & $466.0 \pm 27$ & $0.74 \pm 0.03$ & $0.18^{a}-0.47^{b}[13,14]$ & $8.02 \pm 0.32$ & $11.4[12]$ \\
$\mathrm{C} 1$ & $466.0 \pm 37$ & $0.78 \pm 0.03$ & - & $12.79 \pm 0.57$ & - \\
$\mathrm{C} 2$ & $480.0 \pm 29$ & $0.53 \pm 0.02$ & - & $6.27 \pm 0.25$ & - \\
$\mathrm{C} 3$ & $376.0 \pm 31$ & $0.51 \pm 0.02$ & - & $4.50 \pm 0.20$ & - \\
$\mathrm{C} 4$ & $414.0 \pm 38$ & $0.60 \pm 0.024$ & - & $5.10 \pm 0.27$ & - \\
$\mathrm{Re}$ & $267.0 \pm 29$ & $0.25 \pm 0.01$ & $0.17[15]$ & $16.46 \pm 0.74$ & $20-60[16]$ \\
$\mathrm{Io}$ & $365.0 \pm 35$ & $0.48 \pm 0.02$ & $0.23[12,15]$ & $9.68 \pm 0.38$ & $10-17[17]$ \\
$\mathrm{Am}$ & $384.0 \pm 38$ & $1.60 \pm 0.06$ & $0.96[12]$ & $34.08 \pm 0.20$ & $22-28[16]$ \\
\hline
\end{tabular}

${ }^{a}$ Corresponds to dentine.

${ }^{b}$ Corresponds to enamel. 


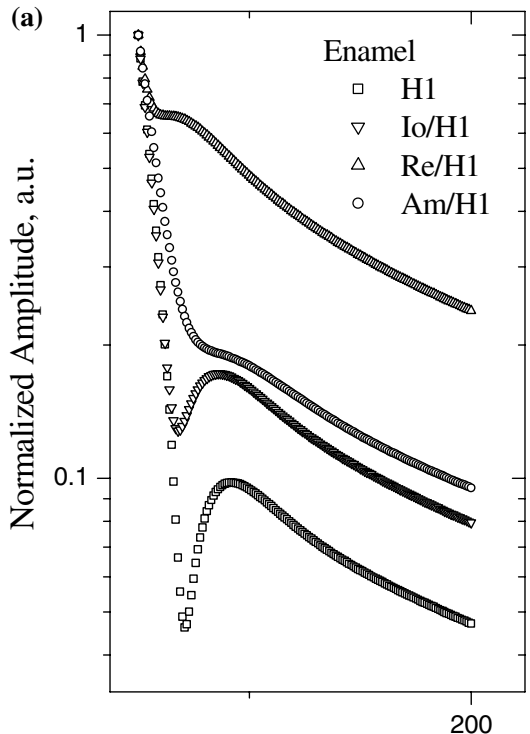

Frequency, $\mathrm{Hz}$

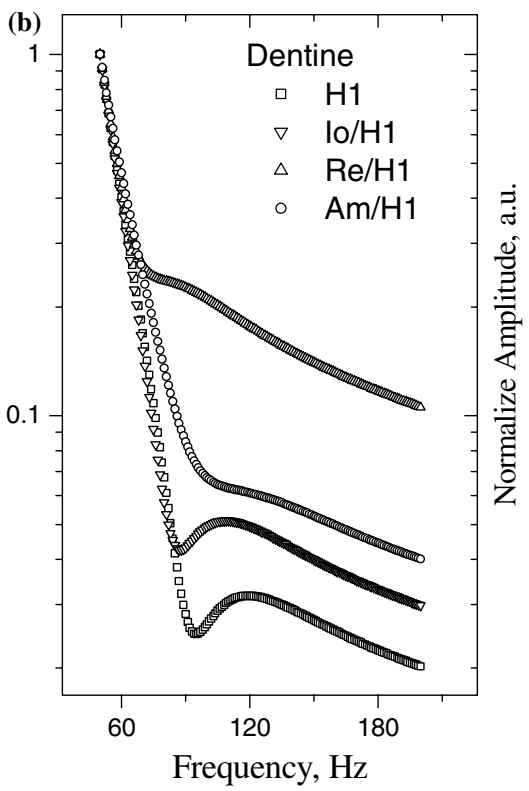

Fig. 4. Amplitude of the PA signal (theoretical results) for teeth (enamel and dentin) and two-layer system composed of teeth and a restorative material (Am, Re, and Io).

As can be seen in Fig. 4, the theoretical PA amplitude shows a resonance, which is due to the presence of both phenomena with a significant phase difference. This resonance changes in the case of the two-layer system. In order to explain this resonance, Eq. (2) can be rewritten as the summation of both complex contributions as follows:

$$
\begin{aligned}
\delta P & =\delta P_{\text {Diff }}+\delta P_{\text {Therm }} \\
& =A_{\text {Diff }} e^{j \phi}+A_{\text {Therm }} e^{j \theta},
\end{aligned}
$$

where $\phi$ and $\theta$ represent phases of the diffusion and thermoelastic contributions, respectively, with the amplitude of this complex function given by

$$
A=\sqrt{A_{\text {Diff }}^{2}+A_{\text {Therm }}^{2}+2 A_{\text {Therm }} A_{\text {Diff }} \cos (\phi-\theta)}
$$

Equation (3) shows that the amplitude $\delta P$ depends not only on the magnitudes of the thermal diffusion and thermoelastic contributions, but also on the phase difference of each mechanism. When the phase difference is equal to $\mathrm{n} \pi$, the expression for the amplitude is $A=$ $\sqrt{A_{\text {Diff }}^{2}+A_{\text {Therm }}^{2} \pm 2 A_{\text {Therm }} A_{\text {Diff }}}$; this corresponds to a change in the slope 
of the amplitude. The resonance is basically due to the coupling between diffusion and thermoelastic vibration mechanisms represented in this expression by the phase-lag difference. In the case of the two-layer system, the analysis of the signal is more complicated since thermal matching accounted for the well known parameter of the thermal reflection coefficient, $\eta=\frac{1-s}{1+s}$, which introduces a new phase lag. We suppose that this new phase lag introduced by the interface of a two-layer system is responsible for a shift in the resonance. Figure 4 shows such a resonance shift for teeth and the restorative dental material obtained by theoretical simulation.

\section{CONCLUSIONS}

The thermal and thermo-mechanical properties of teeth and restorative materials have been studied using the OPC technique. These properties were measured simultaneously at room temperature, using a minimum amount of material. The errors obtained for the measured values are less than $5 \%$ for the thermal diffusivity. These errors were obtained from the data fit analysis. Comparisons with literature results show reasonable agreement in magnitude, and some discrepancies could be explained by the differences in sample preparation, as was discussed in Section 3. Based on our experimental results, theoretical simulations of the photothermal effects in the two-layer system (formed by restorative material and teeth) have been performed. These results show that the OPC technique can be used to study the thermal compatibility of dental material with the restorative materials. Theoretical analysis of the resonance found that the amplitude may be used to characterize the two-layer system; however, further experimental studies should be performed in order to verify the theoretical results. Measurements at higher temperatures (no more than $50^{\circ} \mathrm{C}$ ) could be of interest in clinical application, if the thermal and thermo-mechanical parameters change significantly.

In summary, the OPC technique is proposed to study teeth and restorative materials ex vivo and theoretical simulation could be used to make predictions of the thermal and thermo-mechanical behavior of the teeth-restorative material system.

\section{ACKNOWLEDGMENTS}

This work was partially supported by CONACyT under Grant No 42891-F, and by CONCyTEG under Grant No 03-04-K118-039 and No 03-16-K117-029. The authors thank Jose Luis Flores and Jose de la Luz Hurtado for their technical assistance in sample preparation. 


\section{REFERENCES}

1. S. J. Bennett, J. Phys. E: Sci. Instrum. 10:525 (1977).

2. S. Battaglia and F. Mango, J. Mater. Sci. Forum 443-444:151 (2004).

3. Y. S. Touloukian, Thermophysical Properties of Matter, Vol. 12, (IFI/Plenum, New York, Washington, 1973), pp. 17a-25a.

4. Y. S. Touloukian, Thermophysical Properties of Matter, Vol. 10, (IFI/Plenum, New York, Washington, 1973), pp. 2-3.

5. A. M. Mansanares, A. C. Bento, H. Vargas, N. F. Leite, and L. C. M. Miranda, Phys. Rev. B. 42:4477 (1990).

6. M. V. Marquezini, N. Cella, A. M. Mansanares, H. Vargas, and L. C. M. Miranda, Meas. Sci. Technol. 2:396 (1991).

7. Nibu A. George and R. Vinayakrishnan, J. Phys.: Condens. Matter 14:4509 (2002).

8. Jyotsna Ravi, M. K. Jayaraj, K. A. Vanaja, K. P. R. Nair, and T. M. A. Rasheed, Semicond. Sci. Technol. 18:693 (2003).

9. J. L. Pichardo and J. J. Alvarado-Gil, J. Appl. Phys. 95:6450 (2004).

10. G. Rousset and F. Lepoutre, J. Appl. Phys. 54:2383 (1983).

11. D. E. Grenoble and J. L. Katz, J. Biomed. Mater. Res. 5:489 (1971).

12. http://www.lib.umich.edu/libhome/Dentistry.lib/Dental_tables/Coefthermexp.html

13. W. S. Brown, W. A. Dewey, and H. R. Jacobs, J. Dent. Res. 49:752 (1970).

14. Y. Fukase, M. Saitoh, M. Kaketani, M. Ohashi, and M. Nishiyama, Dent. Mater. J. 11:189 (1992).

15. W. A. Brantley and R. E. Kerby, J. Oral Rehabil. 20:61 (1993).

16. J. M. Powers, R. W. Hostetler, and J. B. Dennison, J. Dent. Res. 58:584 (1979).

17. http://www.21 cecpharm.com/chem/pl0020.htm 